\title{
A Comment on Fundamental Geodetic Constants
}

\author{
by Erwin Groten*
}

(1) Velocity of light in vacuo:

$c=299792458 \mathrm{~ms}^{-1}$ (defining constant)

(2) Newtonian gravitational constant:

$\mathrm{G}=(6.67259 \pm 0.00030) \cdot 10^{-11} \mathrm{~m}^{3} \mathrm{~s}^{-2} \mathrm{~kg}^{-1}$

(3) Geocentric gravitational constant

(incl. mass of atm.):

$\mathrm{GM}=(398600.4415 \pm 0.0008) \cdot 10^{9} \mathrm{~m}^{3} \mathrm{~s}^{-2}$

(in TDB):

$\mathrm{GM}=398600.4356 \cdot 10^{9} \mathrm{~m}^{3} \mathrm{~s}^{-2}$

(4) Mean angular velocity of Earth's rotation : $\quad \omega=7292115 \cdot 10^{-11} \mathrm{rad} \mathrm{s}^{-1}$ (rounded)

(5) Zonal second-degree geopotential parameter (tide-free):

(incl. zero-frequency tide):

$\mathrm{J}_{2}=(1082.6269 \pm 0.0006) \cdot 10^{-6}$

$\mathrm{J}_{2}=1082.6362 \cdot 10^{-6}$

(6) Geopotential at the geoid:

$\mathrm{W}^{0}=(62636857.5 \pm 1.0) \mathrm{m}^{2} \mathrm{~s}^{-2}$ (Bursa et al.) $W^{0}=(62636856.26 \pm 1.0) \mathrm{m}^{2} \mathrm{~s}^{-2}$ (Rapp)

Geopotential scale factor: $\left.\mathrm{R}_{0}=\mathrm{W}^{0} / \mathrm{GM}=6363672.40 \pm 0.1\right) \mathrm{m}$

(7) Mean equatorial radius of Earth's ellipsoid:

$\mathrm{a}=(6378136.55 \pm 0.1) \mathrm{m}$

(8) Parameter $\mathrm{H}=(\mathrm{C}-(\mathrm{A}+\mathrm{B}) / 2)$ in precession constant:

H $=0.0032729567 \pm 2 \cdot 10^{-9}$

(9) Mean equatorial gravity:

$\mathrm{g}_{\mathrm{e}}=(978032.74 \pm 0.08) \cdot 10^{-5} \mathrm{~m} \mathrm{~s}^{-2}$

(10) Earth's polar flattening (tide-free):

$1 / f=298.258 \pm 0.001$ (with indirect zero-frequency tide incl.):

$1 / \mathrm{f}=298.257 \pm 0.001$

(11) Factor of potential of centrifugal force:

(12) Equatorial flattening:

$q=(3461.390 \pm 0.002) \cdot 10^{-6}$

$1 / f_{1}=91500 \pm 100$

(13) Longitude of semi-major axis of equatorial ellipse:

$\lambda_{1}=-(14.95 \pm 0.05)^{\circ}$

(14) Moments of inertia of Earth (tide free):

$A=(8.0094 \pm 0.0003) \cdot 10^{37} \mathrm{~kg} \mathrm{~m}^{2}$

$B=(8.0096 \pm 0.0003) \cdot 10^{37} \mathrm{~kg} \mathrm{~m}^{2}$

$\mathrm{C}=(8.0358 \pm 0.0003) \cdot 10^{37} \mathrm{~kg} \mathrm{~m}{ }^{2}$

$\mathrm{Ma}^{2}=(2.4301 \pm 0.0001) \cdot 10^{38} \mathrm{~kg} \mathrm{~m}{ }^{2}$

(15) Temporal change of $\mathrm{J}_{2}$ :

$\dot{\mathrm{j}}_{2}=-(2.6 \pm 0.3) \cdot 10^{-9} \mathrm{cy}^{-1}$

(16) Lunar tidal acceleration:

$\dot{n}=-(26.3 \pm 0.5) " \mathrm{cy}^{-2}$ (general)

$\dot{n}=-(25.9 \pm 0.5) " \mathrm{cy}^{-2}$ (for JPL-model)

$\dot{n}$ (semidiurnal) $=-(22.4 \pm 0.6) " \mathrm{cy}^{-2}$

$\dot{\omega}=-(5.0 \pm 0.3) \cdot 10^{-22} \mathrm{rad} \mathrm{s}^{-2}$

(17) Long-term total deceleration (rounded):

$\dot{\omega}_{\text {rel. }}=+(1.29 \pm 0.28) \cdot 10^{-22} \mathrm{rad} \mathrm{s}^{-2}$ (caused by $\dot{\mathbf{J}}_{2}$ (long-term)):

presented at the IAU General Assembly, The Hague, August 1995 